Journal of

Women's Health and Gynecology

\title{
Agreement Conform Current Operational Rules and Directives (ACCORD): A Novel Tool to Reach Multidisciplinary Consensus
}

Stephanie M.P. Lemmens ${ }^{1}$, Hubertina C.J. Scheepers ${ }^{2}$, Veronica A. Lopes van Balen ${ }^{3}$, Yvonne C.M. Röselaers ${ }^{4}$ Raymond G. De Vries ${ }^{5}$, Marc E.A. Spaanderman ${ }^{2}$

${ }^{1}$ Department of Obstetrics and Gynecology, GROW School for Oncology and Developmental Biology, Maastricht University, Universiteitssingel 40, 6229 ER Maastricht, The Netherlands

${ }^{2}$ Department of Obstetrics and Gynecology, Maastricht University Medical Center, Postbus 5800, 6202 AZ Maastricht, The Netherlands

${ }^{3}$ Department of Obstetrics and Gynecology, Maxima Medical Centre, Postbus 7777, $5500 \mathrm{MB}$ Veldhoven, The Netherlands. ${ }^{4}$ ROS Robuust, Lichttoren 32 (Igluu), 5611 BJ Eindhoven, The Netherlands

${ }^{5}$ Research Center for Midwifery Science Maastricht, Zuyd University, Maastricht, The Netherlands; Center for Bioethics and Social Sciences in Medicine, University of Michigan Medical School. Ann Arbor, Michigan

${ }^{*}$ Corresponding author: Stéphanie Lemmens MD, Department of Obstetrics and Gynaecology, GROW School for Oncology and Developmental Biology, Maastricht University, PO Box 5800, 6202 AZ Maastricht, Tel: +31 43387 6543; E-mail:stephanie. lemmens@maastrichtuniversity.nl

Received Date: August 15, 2019 Accepted Date: September 30, 2019 Published Date: October 03, 2019

Citation: Stephanie M.P. Lemmens (2019) Agreement Conform Current Operational Rules and Directives (ACCORD): A Novel Tool to Reach Multidisciplinary Consensus. J Womens Health Gyn 5: 1-11.

\begin{abstract}
Objective: Collaboration between healthcare professionals with overlapping expertise but fundamental different views and perspectives requires consensus on best care practices. The aim of the current study is to evaluate a novel model to reach fast, concrete and acceptable agreement.
\end{abstract}

Study design: The Agreement Conform Current Operational Rules and Directives-tool (ACCORD) is developed to promote collaboration between independent midwives $(\mathrm{M})$ in primary care, and gynecologists-obstetricians $(\mathrm{G})$ working in secondary and tertiary care. The ACCORD-tool consists of a four-step, bottom-up approach: first, summarize the current evidence, second translate the evidence into statements, third send online surveys to all caregivers $(n=137)$ in the work field to rank these statements by their level of agreement, and finally, review the statements with a team of representatives $(n=23)$ to reach consensus. Statements are directly accepted or rejected when the agreement is high (exceeding 8) or low (below 3) on a scale 1-10. Statements are discussed when scores are neutral (between 3 and 8 ), or have a broad range in opinion (SD $>2$ ). Outcome measures are the completion of a consensus document within the chosen time frame and the level of acceptance by the participants of both the consensus document and the ACCORD-tool and its influencing factors.

Results: After discussion of $75 \%$ of statements, the consensus rate was $92 \%$. A document with recommendations for the entire field of obstetric care was finished within the chosen period of two years. Participants' satisfaction expressed in 'recommending the tool' and 'being pleased with the final document' differs between collaborating professions $(\mathrm{M}=5.6 \pm 2.4 \mathrm{vs}$ $\mathrm{G}=7.9 \pm 1.4, \mathrm{p}<0.01$ and $\mathrm{M}=6.1 \pm 1.9$ vs $\mathrm{G}=8.3 \pm 0.9, \mathrm{p}<0.01$, on a $1-10$ scale, respectively). Satisfaction is positively influenced by the information supplied on scope, purpose, and method before and during the process and the extent to which everyone's opinion is considered. A feeling of power imbalance and loss of autonomy negatively affect participants' satisfaction.

C2019 The Authors. Published by the JScholar under the terms of the Creative Commons Attribution License http://creativecommons.org/licenses/ by/3.0/, which permits unrestricted use, provided the original author and source are credited. 
Conclusion: The ACCORD-tool is a promising approach for developing collaborative inter- professional schedules with moderate-to-good acceptance by participants, within a limited time frame.

Keywords: multidisciplinary; bottom-up; consensus

\section{Background}

The contemporary practice of medicine is increasingly relying on multidisciplinary collaboration, characterized by a situation in which different professionals work on the same project but independently, parallel or sequential [1]. Multidisciplinary collaboration is commonly defined by five underlying pillars $[1,2]$. First, responsibilities need to be shared to cooperate constructively. Second, a partnership needs to occur based on mutual trust and respect, valuing the contributions and perspectives of the other professionals. Third, equal partnership calls for interdependent rather than an autonomous collaboration. Fourth, individuals participating in a multidisciplinary collabortion need to recognize each other's professional capacities based on their knowledge and experience. Finally, a good collaboration is a constantly developing, dynamic and interactive process [1]. Collaborating across disciplines requires agreement on best care practices within a team consisting of members with different training, guidelines and perspectives. It is thought that a judgment that integrates the expertise of several professionals results in better decisions $[3,4]$. Participants collaborating in a multidisciplinary team must come to consensus coping with problematic issues as concerns about best practices, autonomy, equal influence, and income. Group decisions made by consensus aim to result in decisions that are satisfactory to all group members. A process that includes the input of all participants and listens to all of their concerns, generates more agreement with the final decision and greater cooperation in implementation $[3,5]$. A common known technique to reach consensus in a small group of people is the Delphi method. The Delphi technique uses panel members that are selected experts in the field that have no direct face-to-face contact and remain anonymous to each other [4]. The Delphi method recommends 10-18 experts on a panel and uses the concept of iterative feedback rounds, with the opportunity for panel members to reflect on their previous response [4].

One strength of the Delphi method is its use of expert opinion, but its weakness is that it is a top-down approach. In order to ensure that new protocols will be implemented by a diverse group of professionals, it is necessary to use a bottom-up approach. A bottom-up approach starts with a commitment to meet the needs of professionals who are most affected by the new policy $[6,7]$. Furthermore, it is important to ensure an equal participation of all professionals involved in the project to prevent imbalances, and a meaningful discussion of content based on current evidence without discussions lead by emotions and personal interests. To this end, we developed a new model to reach concrete and acceptable management agreement within a reasonable period of time. This paper describes the evaluation of this model on both effectiveness defined as the level of consensus on content and a process evaluation defined as the satisfaction of the participants and factors influencing this satisfaction. The level of consensus needs to be high in order to have a successful implementation of the decisions made. The evaluation of the process is important to investigate factors influencing participant's satisfaction and to improve the the decision-making process in the future.

\section{Methods}

This novel model was named the Agreement Confirm Current Operational Rules and Directives-tool (ACCORD). The ACCORD-tool had to meet three important criteria. First, the tool had to be acceptable to those using it. The participating professionals discussed current professional guidelines and protocols, thereby weighing their opinions and concerns, to reach consensus on the content of care for clinical practice.

Second, the tool had to be concrete, with recommendations based on scientific evidence and decisions that were directly and conclusively applicable in daily practice. In addition, the opinions of participants were weighed and presented objectively (using means and standard deviations, SD), to prevent agreements solely based on emotions, self-interest, and strong but unfounded opinions. Decisions were agreed upon when at least $85 \%$ of the team was in favor. Third, the process had to be fast with consensus on two main topics every three months. The ACCORD-tool which complies with the above-mentioned criteria consists of four steps to reach consensus (figure 1). In a chosen period of 2 years, acceptable and practical professional choices and actions were assessed, discussed and determined according to these four steps. The first step involves a summary of existing evidence for each subject selected, performed by the researcher of the project group. This overview of the current knowledge and guidelines is sent to the participants of the team before the team meeting. The second step includes the creation of a flowchart, based on data extracted from literature, specifying the options on which a decision should be taken. In the third step these decision points are translated into statements and all participants are invited to rank each statement in an online survey by the 
level of agreement on a 1 (totally disagree) to 10 (totally agree) Likert-scale (Survey Monkey, Palo, Alto, CA, USA). By using a ranking scale we are able to assess the opinions of participants in a more or less objective manner, using means and SDs. The survey givesparticipants the opportunity to express any concerns regarding a topic in text boxes added to each statement. In the fourth step survey results are presented and discussed by a team of professionals representing their peers in an effort to reach consensus. Statements with a mean between 3 and 8 , or a SD $>2$ must be discussed, a mean below 3 or above 8 and a SD $<2$ leads to immediate rejection or acceptance respectively. The final document, containing all agreements, is intended to serve as a guiding document to support professionals in their daily practice. It is not meant to substitute for clinical judgement. In the example, suspected fetal growth restriction is further elaborated, but steps one to four were applied to all subjects. We evaluated the tool by assessment of the level of consensus and the satisfaction of the participants, measured with an online survey (see results - a process evaluation).

\section{STEP 1 Summary of the literature based on (inter)national guidelines and protocols}

\section{STEP 2 Flowchart defining decision points and guiding questions}

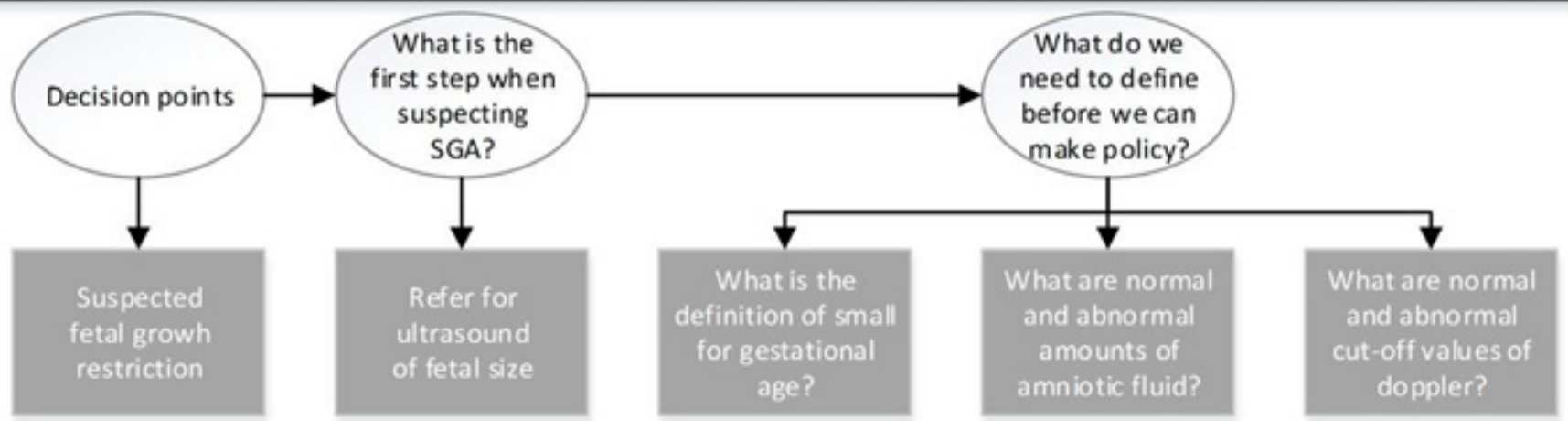

\section{STEP 3 Online survey: ranking of statements by professionals}

The cut-off value for defining a fetus small-for-gestational-age (SGA) is the $10^{\text {th }}$ percentile.

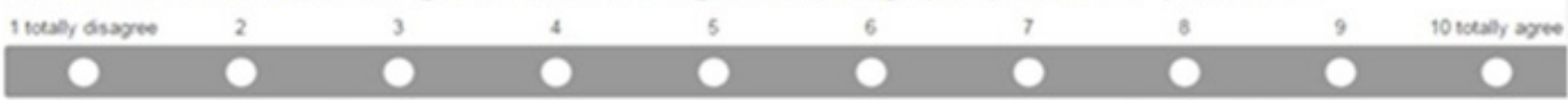

The cut-off value for defining a fetus small-for-gestational-age (SGA) is the $5^{\text {th }}$ percentile.

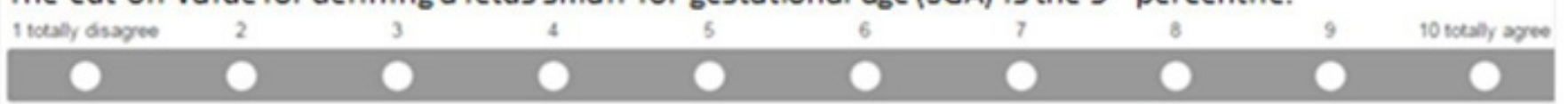

The cut-off value for defining a fetus small-for-gestational-age (SGA) is the $2.3^{\text {th }}$ percentile.

\begin{tabular}{|c|c|c|c|c|c|c|c|c|c|}
\hline 1 tocesly dasgee & 2 & 3 & 4 & 5 & 6 & 7 & 8 & 9 & 10 tecesely aye \\
\hline 0 & 0 & 0 & 0 & 0 & 0 & 0 & 0 & 0 & 0 \\
\hline
\end{tabular}

STEP 4 Discussion in team sessions based on statement scores (mean \pm SD) to reach consensus

\begin{tabular}{lll}
\hline $\begin{array}{l}\text { Statement results } \\
\begin{array}{l}\text { The cut-off value for defining a fetus small-for-gestational-age (SGA) is the } \\
10^{\text {th }} \text { percentile. }\end{array}\end{array}$ & $\begin{array}{l}\text { Team } \\
\text { mean } \\
\text { (SD) }\end{array}$ & $\begin{array}{l}\text { Peers } \\
\text { mean } \\
\text { (SD) }\end{array}$ \\
\hline $\begin{array}{l}\text { The cut-off value for defining a fetus small-for-gestational-age (SGA) is the } \\
5^{\text {th }} \text { percentile. }\end{array}$ & $5.8(2.8)$ & $6.3(3.0)$ \\
\hline $\begin{array}{l}\text { The cut-off value for defining a fetus small-for-gestational-age (SGA) is the } \\
2.3^{\text {th }} \text { percentile. }\end{array}$ & $4.6(1.8)$ & $4.9(2.1)$ \\
\hline
\end{tabular}

Figure 1, Four step ACCORD method, ${ }^{*}$ SGA: small for gestational age, as to illustrate. 


\section{Participants}

The organizational structure of the ACCORD-tool consists of three groups: 1) a project group, consisting of a gynecologist, an independent midwife, a researcher who worked as a resident in gynecology and a former clinical working midwife. Together they were responsible for the organizational process, preparation of the meetings (summarize the literature, send the survey etcetera.), structuring the meetings and documentation of the decisions made, 2) one or more team(s) with representatives are chosen and mandated by their peers to take final decisions during team meetings and 3) a group of peers, all professionals in the field which were invited to give their opinion on obstetric topics in the surveys (figure 2). Professionals of different disciplines or echelons within disciplines should be invited to participate. All participants' email addresses were obtained and a declaration of collaboration was signed in which the participants agreed to comply with the rules, including principles for teamwork, mutual respect, and communication (AppendixI).

\section{Statistics}

Data are expressed as mean with standard deviation and analyzed parametrically. Logistic regression was used to calculate odds ratios to analyze associations with satisfaction with the tool. Multivariate analysis within the domains of audited associates (representation, participation, tool, and profession) was used to determine the key components of these associates. Spearman's correlation was used to analyze the relationship between audited associates and satisfaction (recommendation of the tooland pleased with the final document, yes $=$ score $>6$ on $1-10$ Likert scale). A two-sided p-value $<0.05$ was considered statistically significant. Statistical analysis was performed using IBM SPSS Statistics 21 .

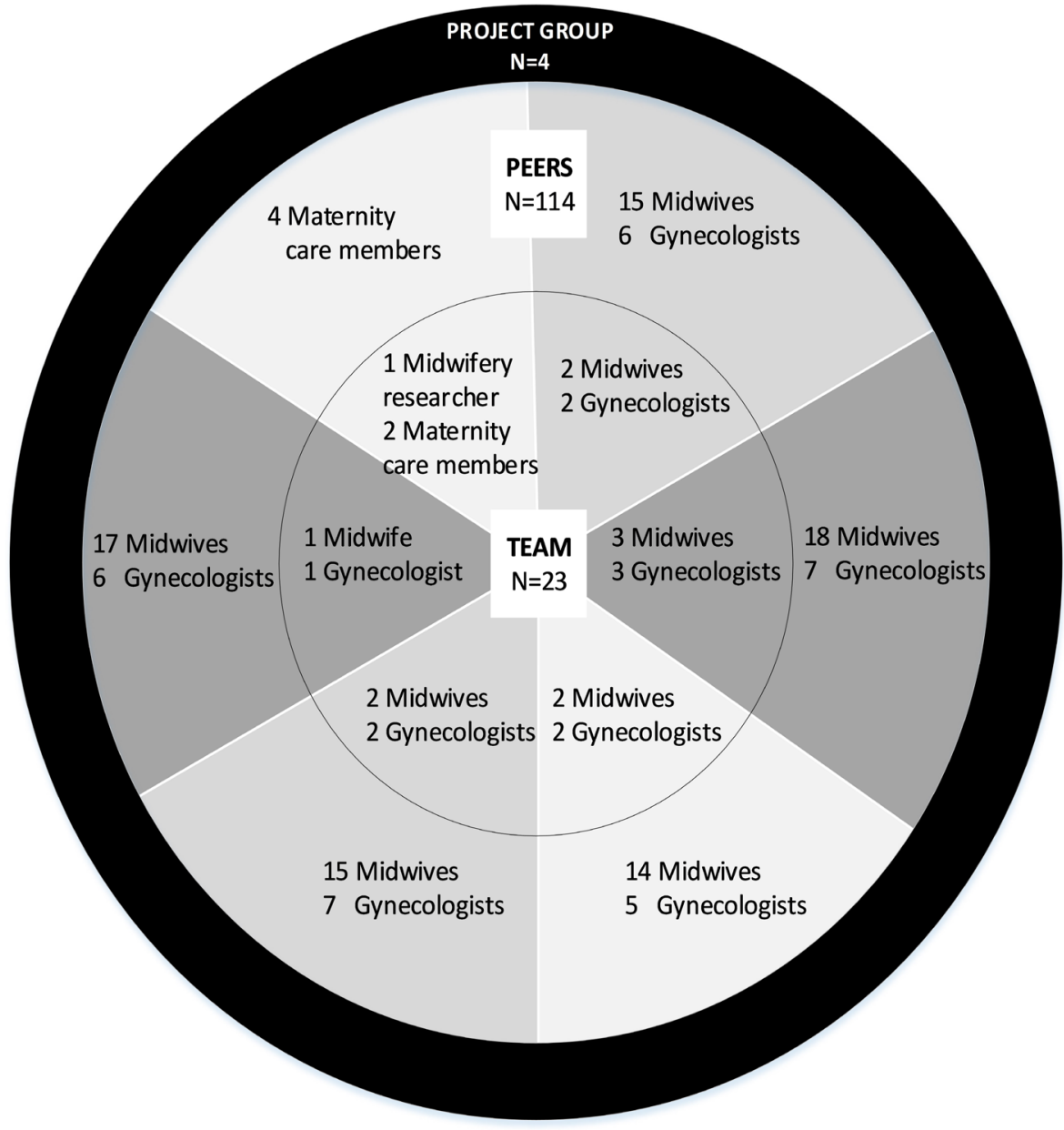

Figure 2: Participants are divided in a group of peers and a team of representatives.

Appendix 1: Required (personal) characteristics of team members and required team conditions. (uploaded on request) 


\section{Results}

We included all 141 professional participants that worked in primary, secondary or tertiary obstetric care between 2013 and 2016 within the catchment area of interest. The coordinating project group $(n=4)$ consisted of obstetric professionals, each with their own expertise. Two teams of representatives $(n=23)$ and a group of peers $(n=114)$ consisted of gynecologists, independent midwives and maternity care members. In total 14 surveys were sent with 507 guiding questions answered by ranking 681 statements and covering 19 main subjects (figure 3 ). The overall response rate was $85 \%$ for the team and $37 \%$ for their peers. Based on the mean and SD, 15\% of all statements were directly accepted, $2 \%$ were immediately rejected and the teams decided $8 \%$ be inconclusive due to indistinct formulation. The vast majority of statements (75\%) had to be discussed which resulted in acceptance (29\%), acceptance in a modified form (19\%) in response to new information emerged during the discussion, and rejection $(27 \%)$ of statements. Consensus was reached on $92 \%$ of statements $(15 \%+29 \%+19 \%=63 \%$ accepted, $2 \%+27 \%$ $=29 \%$ rejected $)$ and $98 \%$ of all guiding questions were answered. For 3 out of 19 subjects, new statements were formulated and two instead of one team meeting were needed to take final decisions.
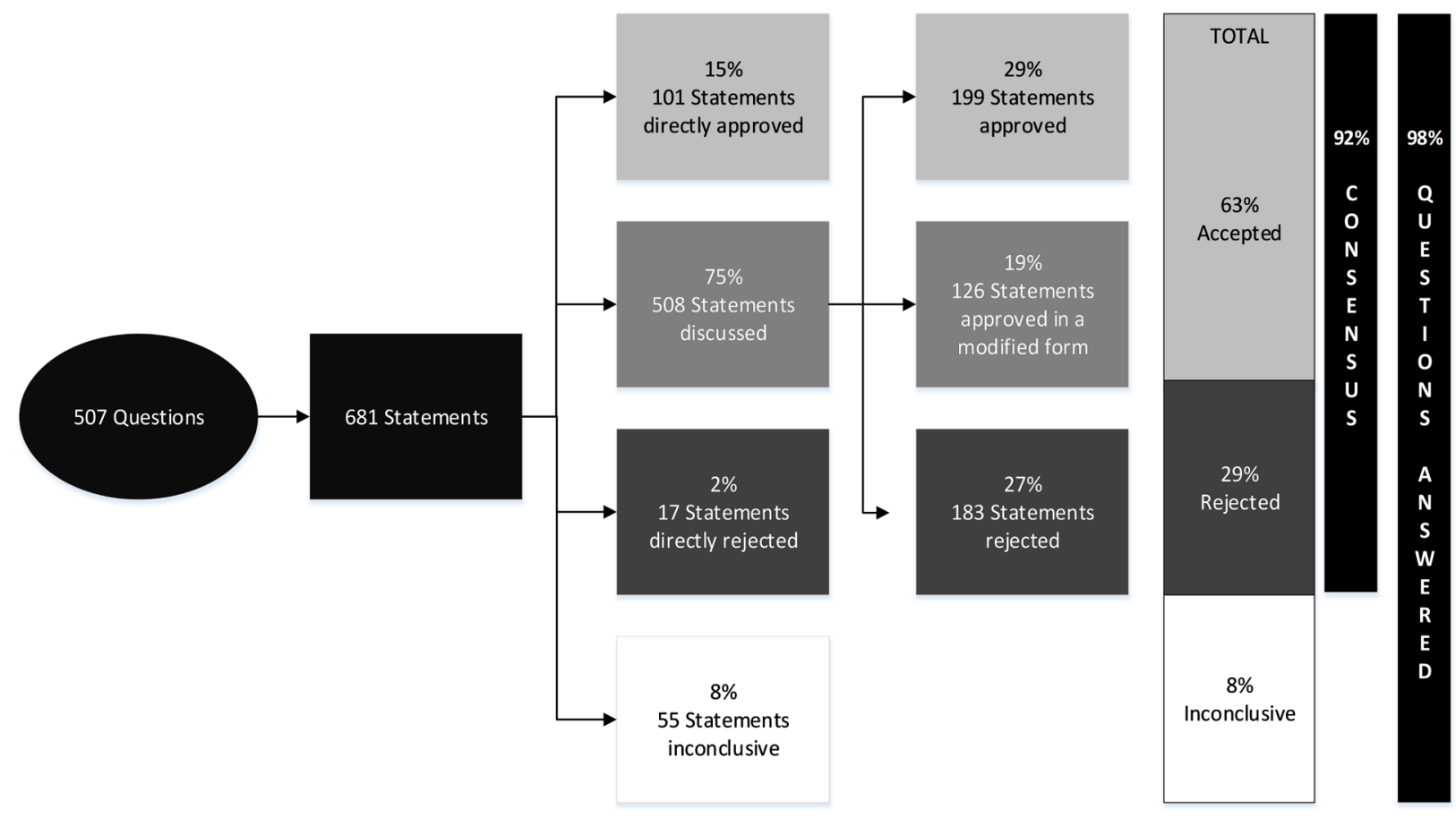

Figure 3: Flowchart of the statement distribution.

\section{Process evaluation}

For gynecologists and midwives, the mean and SD for each statement are shown in Table 1 . For the two main questions, regarding recommending the ACCORD-tool (M 5.6 \pm 2.4 vs $G 7.9 \pm 1.4, \mathrm{p}<0.01)$ and the satisfaction with the final document ( $\mathrm{M} 6.1 \pm 1.9$ vs $\mathrm{G} 8.3 \pm 0.9, \mathrm{p}<0.01$ ), significant differences are seen with higher mean scores for gynecologists.

Representation - Both professions trusted their representatives (M 8.3 \pm 1.4 vs $\mathrm{G} 10.0 \pm 0.0, \mathrm{p}<0.01$ ), and were satisfied with the way the representatives informed them about the progress $(M$ $7.2 \pm 2.1$ vs $G 9.7 \pm 0.6, p=0.05)$ and the decisions made by the team (M 7.2 \pm 2.3 vs $\mathrm{G} 9.7 \pm 0.6, \mathrm{p}=0.07)$.
Participation - Gynecologists had significantly higher mean scores, for questions regarding the weighing of their opinion during the process (M 5.9 \pm 2.2 vs $G 8.7 \pm 0.9, \mathrm{p}<0.01$ ) and respecting the opinion of the region during the team meetings ( $M$ $5.9 \pm 2.3$ vs $\mathrm{G} 8.1 \pm 1.9, \mathrm{p}<0.01)$. Midwives may have felt a certain degree of a power imbalance (M 6.3 \pm 2.2 vs $G 4.2 \pm 3.1, p=0.07$ ), although not significantly different compared to gynecologists. The factor 'not taken seriously' (M 5.0 \pm 2.7 vs G 2.6 $\pm 2.1, \mathrm{p}<0.05$ ) had a low mean score for both professions, but, compared to gynecologists midwives felt they were taken less seriously.

Tool - Midwives judged the amount of information they received prior to the project to be less sufficient (M 5.4 \pm 2.2 vs G $8.0 \pm 1.6$, $\mathrm{p}<0.01)$ as compared to gynecologists. Midwives were also more 


\begin{tabular}{|c|c|c|c|c|c|}
\hline \multirow[t]{2}{*}{ Question } & \multicolumn{2}{|c|}{$\begin{array}{l}\text { Midwives } \\
\text { TOTAL } n=43\end{array}$} & \multicolumn{2}{|c|}{ Gynaecologists TOTAL $n=10$} & \multirow[b]{2}{*}{$p$-value } \\
\hline & Mean & SD & Mean & SD & \\
\hline \multicolumn{6}{|l|}{ Representation } \\
\hline $\begin{array}{l}\text { I trusted my representative totally in } \\
\text { decision making. }\end{array}$ & 8.3 & 1.4 & 10.0 & 0.0 & $<0.01$ \\
\hline $\begin{array}{l}\text { The representative in my region has } \\
\text { informed me sufficiently during the } \\
\text { process on the progress of the } \\
\text { developed care pathways. }\end{array}$ & 7.2 & 2.1 & 9.7 & 0.6 & 0.05 \\
\hline $\begin{array}{l}\text { The representative in my region has } \\
\text { informed me sufficiently during the }\end{array}$ & 7.2 & 2.3 & 9.7 & 0.6 & 0.07 \\
\hline
\end{tabular}

\section{Participation}

My opinion is sufficiently taken into

5.9

2.2

8.7

0.9

$<0.01$

account during the process.

The view of my region was respected

$\begin{array}{lll}5.9 & 2.3 \quad 8.1\end{array}$

1.9

$<0.05$

during the team meetings.

I was not taken seriously.

5.0

2.7

2.6

2.1

$<0.05$

l experienced a power imbalance.

$\begin{array}{lll}6.3 & 2.2 & 4.2\end{array}$

3.1

0.07

\section{Tool}

I received sufficient information before

5.4

2.2

8.0

1.6

$<0.01$

the start of the project to know what

was going to happen.

The opinion of all participants was

6.8

$2.5 \quad 8.6$

0.8

$<0.01$

asked.

The results were presented objectively.

$\begin{array}{lll}6.6 & 2.5 \quad 8.6\end{array}$

$<0.01$

The opinion of all participants was

$\begin{array}{lll}6.1 & 2.7 & 8.4\end{array}$

1.4

$<0.01$

weighted in the process.

\section{Profession}

I experienced financial consequences.

5.6

2.8

5.5

2.5

0.91

l experienced a difference in view.

7.9

1.6

8.3

1.1

0.42

l experienced a difference in expertise.

$\begin{array}{lll}6.7 & 1.7 & 7.6\end{array}$

1.6

0.13

I experienced loss of autonomy.

$\begin{array}{lll}6.8 & 2.3 & 4.6\end{array}$

2.5

0.01

\section{Other}

I would recommend the used tool to others.

$\begin{array}{lll}5.6 & 2.4 & 7.9\end{array}$

1.4

$<0.01$

I am pleased with the final document.

$\begin{array}{lll}6.1 & 1.9 & 8.3\end{array}$

0.9

$<0.01$

Table 1: Statement means and SD for midwives and gynecologists. 
likely to be unaware of the impact the project would have on their daily practice. Both professions reported three factors of positive influence, although in each case scores were lower for midwives: 1) all participants were asked to give their opinions ( $\mathrm{M} 6.8 \pm 2.5$ vs $G 8.6 \pm 0.8, p<0.01), 2$ ) the results were presented objectively (M 6.6 \pm 2.5 vs $\mathrm{G} 8.6 \pm 1.0, \mathrm{p}<0.01$ ) and 3) the opinions of all participants were weighed in the process ( $\mathrm{M} 6.1 \pm 2.7$ vs $\mathrm{G} 8.4 \pm 1.4$, $\mathrm{p}<0.01)$.

Profession - Financial consequences (M 5.6 \pm 2.8 vs G 5.5 \pm 2.5 , $\mathrm{p}=0.91$ ) coming from the chosen decisions were scored neutral. Both professions reported the difference in views $(M 7.9 \pm 1.6$ vs $G 8.3 \pm 1.1, p=0.42$ ) and expertise ( $M$ 6.7 \pm 1.7 vs $G 7.6 \pm 1.6$, $\mathrm{p}=0.13$ ). Considering other influencing factors, midwives scored higher on the loss of autonomy compared to gynecologists ( $\mathrm{M}$ $6.8 \pm 2.3$ vs $\mathrm{G} 4.6 \pm 2.5, \mathrm{p}<0.05)$.

The black boxes in Figure 4 show factors that have a significant effect on recommending the used ACCORD-tool to others and the opinion on the final document. In appendix II all significant correlations are shown. Appendix II: Significant correlations for the two main questions about the final document and the method. Abbreviations: $\mathrm{G}=$ gynecologists, $\mathrm{M}=$ midwives (uploaded on request). Essential for recommending the tool is the information supply during the process by the representative on the progress of the decisions (OR 1.7 (95\%-CI 1.1-2.7)), team meetings in which the view of different regions is respected (OR 2.2 (95\%-CI 1.2-4.2)) and the information supplied prior to the project (OR 2.7 (95\%-CI 1.1-6.3)). If participants reported a power imbalance (OR 0.5 (95\%-CI 0.2-0.8)) or loss of autonomy (OR 0.7 (95\%-CI 0.5-0.9)), they were less likely to recommend the tool. Being pleased with the final document is dependent on the way the opinion of the participants was taken into account during the process (OR 1.8 (95\%-CI 1.0-3.1)) and if the results were considered to be presented objectively (OR 1.9 (95\%-CI 1.2-2.9)). Experiencing a power imbalance (OR 0.3 (95\%-CI 0.1-0.7)) or loss of autonomy (OR 0.7 (95\%-CI 0.5-1.0)) had a negative influence on the opinion of the finalproduct.

\section{Discussion}

By using the ACCORD-tool consensus was reached on $92 \%$ of all statements that were rated. Despite being based on current scientific evidence, three-quarters of all statements were debated before consensus was reached. Our process analysis showed that gynecologists were more satisfied with the process than midwives.

\section{Barriers for collaboration in obstetrics}

During our decision-making process, we faced some challenges we had to overcome. It is known that the Dutch obstetric care system has several substantial barriers to collaboration [8]. Midwives care for healthy pregnant and childbearing women, while gynecologists/obstetricians attend women with complications in pregnancy and childbirth, resulting in fundamentally different perspectives and different views on the course of pregnancy and delivery [9]. Midwives view pregnancy and delivery as healthy, physiological events whereas gynecologists are more prone to be concerned about adverse events. Consequently, gynecologists conduct more checks on the condition of pregnant women and intervene more frequently than midwives [10]. These different views became evident during our team meetings, where the professionals discussed, for instance, the number of check-ups during pregnancy, the need and value of medical interventions, the introduction of new interventions and diagnostic thresholds. The starting point for these discussions were the survey results. The mean and SD for each statement and each profession (gynecologists and midwives) were presented), but also concerns expressed in the survey comments. Besides a different view between gynecologists and midwives, hierarchical structures influenced our decision-making process. In general, healthcare environments are characterized byhierarchical structures, with the specialist on top of the hierarchy [11]. Professionals at the lower end of the hierarchy, tend to be uncomfortable to raise their concerns or bring up the problems their facing [11]. When hierarchical differences come into play, different perceptions on collaboration and communication can diminish collaborative interactions necessary to provide the best care. Research of Van der Lee already showed that although obstetricians express a willingness to cooperate with them, midwives experience a power imbalance in their relationships with their specialist colleagues [8]. Midwives feel that obstetricians see themselves as having a superior role and believe that this perceived power imbalance may arise partially from a different level (university versus vocational) and length of education [12]. Midwives felt they were not taken seriously or trusted, reporting that when they referred a pregnant woman to the hospital, gynecologists would often repeat the evaluation of the woman [8]. From a historical perspective, this feeling of power imbalance can be explainedas a result of the hierarchical structure. Midwives started as autonomous professionals, responsible for all pregnant women, only consulting another caregiver if the child had died during delivery [12]. However, already in the nineteenth century, a new law was 


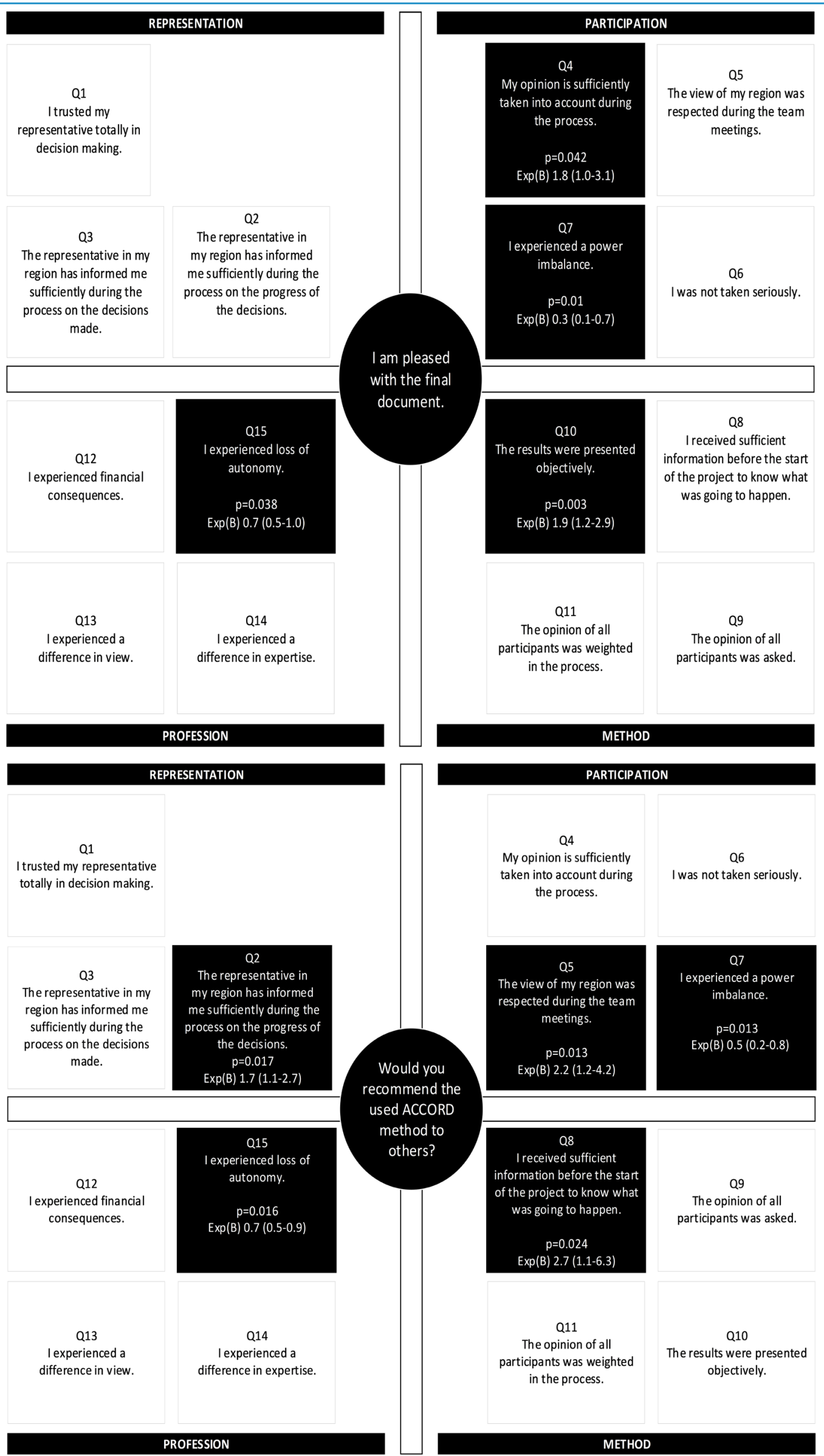

Figure 4: Factors that have a significant effect, marked with the black boxes, on recommending the used ACCORD-method to others and opinion on the final document. 
introduced in The Netherlands, which divided obstetric responsibilities in pathological labor for doctors and physiological labor for midwives, thereby restricting the autonomy of midwives [12]. Evaluating our process, midwives also reported they felt a certain degree of the power imbalance between them and gynecologists, despite the fact that every professional had the opportunity to give their opinion and each opinion counted equally. Probably this is due to the aforementioned historical context. By developing shared protocols using the ACCORD-tool and increasing the collaboration between both professions, we expect that this feeling of power inequality will decrease in future decision-making processes. In addition, separation of the financial structures of both occupational groups could lead to competing financial interests that may put pressure on given care $[9,13]$. Midwives are independent entrepreneurs and changes in obstetric care management can result in loss of income. In our decision-making process, survey contained statements regarding financial consequences to give professionals the opportunity to express any concerns. These concerns were taken into account and discussed during the team meetings. Given the five pillars of successful collaboration, almost all supportive elements were incomplete or absent in our project. Even though these secondary conditions for collaboration were not met, the ACCORD-tool allowed midwives and gynecologists to achieve moderate to good results in reaching consensus. We, therefore, regard the four steps of the ACCORD-tool as a promising approach for reaching consensus in situations where collaboration between stakeholders is mandatory but difficult.

Instead of designing a novel strategywe could have used the top-down Delphi-method [14, 15]. The Delphi technique uses panel members that are selected experts in the field that have no direct face-to-face contact. Two strengths of this technique, because each individual panel member can write his comments, recommendations and solutions to the problem independently and anonymously, unaffected by peer pressure, status or dominant viewpoints of other participants [4, 15-17]. The Delphi method uses the concept of iterative feedback rounds, in which participants are able to revise their opinion after they received feedback, until after some rounds the opinions of the participants stabilize $[4,16,18]$. The use of experts and anonymity is at the same time one of the weaknesses of the Delphi technique, as this is a top-down approach in which the opinion of the people working in the field which are most affected by the decisions is not investigated. This could be a challenge for implementation when the expert opinion does not correspond to the opinion of the professionals in the field. Anonymity may lead to a lack of accountability [17].
In our situation, however, the ACCORD-tool offered certain advantages. Most important, ACCORD allowed faceto-face contact between at least a subgroup of the participants during the team meetings. This face-to-face contact gave the project group the opportunity to clarify uncertainties, discuss the survey results, and give direct feedback to each other. Second, in contrast to the Delphi-method, where only the opinion of the expert panel is weighted, the ACCORD-tool considers the opinion of all participants to be equally important. This bottom-up approach helps to create shared responsibility via an enriched and constructive conversation. If the opinions of the professionals involved are ignored, there is a real risk of a lack of support for decisions made and, as a consequence, a lower likelihood of implementation of the new agreements [19]. This ACCORD-tool is useful in every situation where group decisions have to be reached affecting working-protocols that have to be followed thereafter.

This can be multidisciplinary healthcare teams with specialists, general practitioners and other first line professionals, but also in business organizations for example. Just as many other tools applied in the use of reaching consensus, the ACCORD-tool aims to facilitate collaboration within different professionals groups. Our experience with the ACCORD-tool was that it was able to reach a high degree of consensus within different groups that lacked the ability to cooperate effectively.

\section{Limitations}

A potential limitation of the ACCORD-tool is the labour-intensive character of writing the summary of the guideline-based evidence, preparing the surveys and team sessions by a medically trained researcher. The upside of these labour intensive preparations, however, is that the time investment for participants were limited and team sessions were highly efficient. Our starting point to summarize evidence was extracting information from existing national and international guidelines and local protocols. No systematic search in medical databases was performed, as guidelines and protocols represent topics on which professionals, top-down, have already agreed upon. Due to the fact that guidelines are usually revised every five years, it is possible that we missed evidence based on recent articles. 


\section{Future perspectives}

Important for other professionals who will use the ACCORD-tool is to pay close attention to the way participants receive information at the beginning of the project, and to be aware of possible influencing factors, both positive and negative. The final product is more likely to be appreciated and the tool is more likely to be recommended to others when the views of all participants are respected and negative influencing factors are limited. Moreover, the representatives should be encouraged to act as ambassadors, providing objective information about the deliberations to their peers, especially in cases where decisions were difficult to reach.

\section{Conclusion}

The ACCORD-tool proved to be an efficient approach for collaboratively reaching agreements on the content of care with a moderate-to-good acceptance by participants in a group of professionals with different views and perspectives.

\section{Abbreviations}

ACCORD: Agreement Conform Current Operational Rules and Directives; SD: standard deviation; G: gynecologists; M: midwives; OR: odds ratio; CI: confidence interval

\section{Declarations and acknowledgments}

Ethics approval and consent to participate: not applicable.

Consent to publish: not applicable.

\section{Availability of data and materials}

The datasets used and/or analyzed during the current study are available from the corresponding author on reasonable request.

Competing interests: The authors declare that they have no competing interests.

\section{Authors' contributions}

SL and VL wrote the manuscript. All authors read and approved the final manuscript.

\section{Acknowledgments}

We are grateful and acknowledge the participation of all professionals involved in the project. This research was funded by ZonMW, The Netherlands, project number 209010006.

\section{References}

1. D'Amour D, Ferrada-Videla M, San Martin Rodriguez L, Beaulieu MD (2005) The conceptual basis for interprofessional collaboration: core concepts and theoretical frameworks. J Interprof Care. 19: 116-131.

2. Xyrichis A, Lowton K (2008) What fosters or prevents interprofessional team working in primary and community care? A literature review. International journal of nursing studies. 45: 140-153.

3. Bressen T (2012) Consensus decision-making: what, why, how. ABA Books.

4. Crisp J, Pelletier D, Duffield C, Adams A, Nagy S (1997) The Delphi method? Nursing research. 46: 116-118.

5. Hartnett T (2011) Consensus oriented decision making: New Society Publishers.

6. Meslin EM (2010) The value of using top-down and bottomup approaches for building trust and transparency in biobanking. Public Health Genomics. 13: 207-214.

7. Sabatier PA (2008) Top-Down and Bottom-Up Approaches to Implementation Research: a Critical Analysis and Suggested Synthesis. Journal of Public Policy 6: 21-48.

8. van der Lee N, Driessen EW, Scheele F (2016) How the past influences interprofessional collaboration between obstetricians and midwives in the Netherlands: Findings from a secondary analysis. Journal of Interprofessional Care 30: 71-76.

9. Perdok H, Jans S, Verhoeven C, van Dillen J, Batenburg R, Mol BW, et al. (2016) Opinions of professionals about integrating midwife- and obstetrician-led care in The Netherlands. Midwifery 37: 9-18.

10. Birthplace in England Collaborative G, Brocklehurst P, Hardy P, Hollowell J, Linsell L, Macfarlane A, et al. (2011) Perinatal and maternal outcomes by planned place of birth for healthy women with low risk pregnancies: the Birthplace in England national prospective cohort study. Bmj 343:d7400.

11. Rosenste MODAH (2008) Professional Communication and Team Collaboration. . Patient Safety and Quality: An EvidenceBased Handbook for Nurses Agency for Healthcare Research and Quality (US). 
12. van der Lee N, Driessen EW, Houwaart ES, Caccia NC, Scheele F (2014) An examination of the historical context of interprofessional collaboration in Dutch obstetrical care. J Interprof Care. 28: 123-127.

13. VLN Scholmerich AP, H Ghorashi, AJM Waelput, P Groenewegen, S Denktas (2014) Improving interprofessional coordination in Dutch midwifery and obstetrics: a qualitative study. BMC pregnancy and childbirth.

14. Jones J (1995) Consensus methods for medical and health services research. Bmj 311:5.

15. Lunenburg FC (2011) Decision Making in Organizations International Journal of Management, Business, and Administration 15 .

16. Dodge B, Clark R (1997) Research on the Delphi Technique. Educational Technology 17:3.

17.C. G. (1987) The Delphi technique: a critique. Journal of Advanced Nursing 12: 729-734.

18. Okoli C, Pawlowski SD (2004) The Delphi method as a research tool: an example, design considerations, and applications. Information \& Management 42: 15-29.

19. Cabana MD Why don't physicians follow clinical practice guidelines? JAMA 282: 8.
Submit your manuscript to a JScholar journal and benefit from:

ब Convenient online submission

ब Rigorous peer review

- Immediate publication on acceptance

T Open access: articles freely available online

9 High visibility within the field

- Better discount for your subsequent articles

Submit your manuscript at http://www.jscholaronline.org/submit-manuscript.php 